\title{
Arrow's Theorem in Spatial Environments, METEOR Research memorandum
}

Citation for published version (APA):

Ehlers, L. H., \& Storcken, A. J. A. (2001). Arrow's Theorem in Spatial Environments, METEOR Research memorandum. METEOR, Maastricht University School of Business and Economics. METEOR Research Memorandum No. 015 https://doi.org/10.26481/umamet.2001015

Document status and date:

Published: 01/01/2001

DOI:

10.26481/umamet.2001015

Document Version:

Publisher's PDF, also known as Version of record

\section{Please check the document version of this publication:}

- A submitted manuscript is the version of the article upon submission and before peer-review. There can be important differences between the submitted version and the official published version of record.

People interested in the research are advised to contact the author for the final version of the publication, or visit the DOI to the publisher's website.

- The final author version and the galley proof are versions of the publication after peer review.

- The final published version features the final layout of the paper including the volume, issue and page numbers.

Link to publication

\footnotetext{
General rights rights.

- You may freely distribute the URL identifying the publication in the public portal. please follow below link for the End User Agreement:

www.umlib.nl/taverne-license

Take down policy

If you believe that this document breaches copyright please contact us at:

repository@maastrichtuniversity.nl

providing details and we will investigate your claim.
}

Copyright and moral rights for the publications made accessible in the public portal are retained by the authors and/or other copyright owners and it is a condition of accessing publications that users recognise and abide by the legal requirements associated with these

- Users may download and print one copy of any publication from the public portal for the purpose of private study or research.

- You may not further distribute the material or use it for any profit-making activity or commercial gain

If the publication is distributed under the terms of Article $25 \mathrm{fa}$ of the Dutch Copyright Act, indicated by the "Taverne" license above, 


\title{
Arrow's Theorem in Spatial Environments*
}

\author{
Lars Ehlers ${ }^{\dagger}$ and Ton Storcken ${ }^{\ddagger}$
}

February 2001 (revised August 2001)

\begin{abstract}
In spatial environments we consider social welfare functions satisfying Arrow's requirements, i.e. weak Pareto and independence of irrelevant alternatives. When the policy space is a one-dimensional continuum such a welfare function is determined by a collection of $2^{N}$ strictly quasi-concave preferences and a tie-breaking rule. As a corollary we obtain that when the number of voters is odd, simple majority voting is transitive if and only if each voter's preference is strictly quasi-concave. When the policy space is multi-dimensional we establish Arrow's impossibility theorem. Among others we show that weak Pareto, independence of irrelevant alternatives, and non-dictatorship are inconsistent if the set of alternatives has a non-empty interior and it is compact and convex. Journal of Economic Literature Classification Numbers: D70, D71.
\end{abstract}

\footnotetext{
*We thank John Weymark for suggesting this research problem at the Summer School on Mathematical Models of Individual and Public Choice, UC at Irvine, Irvine, California, U.S.A., July 9-28, 2000. We acknowledge useful comments and suggestions by John Duggan and William Thomson. Part of the paper was written while the first author was visiting the Department of Economics at University of Rochester.

${ }^{\dagger}$ Département de Sciences Économiques and C.R.D.E., Université de Montréal, Montréal, Québec H3C 3J7, Canada; e-mail: lars.ehlers@umontreal.ca

$\ddagger$ Department of Quantitative Economics, Maastricht University, P.O. Box 616, 6200 MD Maastricht, The Netherlands; e-mail: t.storcken@ke.unimaas.nl
} 


\section{Introduction}

A social welfare function is a procedure for aggregating profiles of individual preferences into social orderings. Arrow's theorem shows that it is impossible for a social welfare function to satisfy weak Pareto (if all individuals strictly prefer one alternative to another, then so does society), independence of irrelevant alternatives (the social ranking of two alternatives only depends on the individual rankings of these alternatives), and non-dictatorship when the preference domain is unrestricted. When the set of alternatives is structured, the assumption of unrestricted domain might be unreasonable. One important exception of this kind are spatial environments. In spatial environments, alternatives are points in a Euclidean space of issue positions and individual preferences are continuous, quasi-concave, and have bliss points. Although the assumption of unrestricted domain is unreasonable in economic and spatial environments, it has been shown that Arrow's theorem remains valid in most of these environments. ${ }^{1}$

There is one well-known spatial environment in which Arrow's requirements are consistent. If the number of voters is odd and the policy space is one-dimensional, then simple majority voting is transitive and satisfies weak Pareto, independence of irrelevant alternatives, and anonymity (Black ,1948; Arrow, 1951, 1963). Simple majority voting is an example of an Arrovian welfare function belonging to the following class: if the number of voters is $n$, fix $n-1$ preferences of constant voters, and apply to each profile of individual preferences majority voting over this profile and the $n-1$ fixed voters. However, a characterization of all welfare functions satisfying weak Pareto and independence of irrelevant alternatives was missing. Our paper fills this gap. The class of welfare functions described above, in which the preferences of the fixed voters are strictly quasi-concave, is characterized by weak Pareto, independence of irrelevant alternatives, and anonymity up to a tie-breaking rule. If anonymity is dropped, then instead of $n-1$ fixed preferences we need $2^{N}$ fixed strictly quasi-concave preferences.

A key step in the proof of Arrow's theorem is that decisiveness of a coalition

\footnotetext{
${ }^{1}$ An excellent review of the literature is Le Breton and Weymark (2000a).
} 
spreads from one pair of alternatives to all pairs of alternatives. In proving the previous fact we need to assume that each individual's preference domain is unrestricted. This is the difference between one-dimensional spatial environments and environments with an unrestricted domain. We show that in one-dimensional spatial environments decisiveness of a coalition spreads in the following way: if a coalition is decisive over " $a$ preferred to $b$ " (where $a, b \in \mathbb{R}$ are such that $a<b$ ), then it is decisive over " $a$ preferred to $c$ " for all $c>b$ and over "c preferred to $b$ " for all $a<c<b$. This implies that for each coalition $S$, there is a point $x_{S} \in[-\infty,+\infty]$ such that $S$ is decisive over " $a$ preferred to $b$ " for all $x_{S}<a<b$ and $N \backslash S$ is decisive over "c preferred to $d$ " for all $d<c<x_{S}$. Therefore, when the peaks of $S$ converge to $-\infty$ and the peaks of $N \backslash S$ to $+\infty$, then an Arrovian welfare function chooses a strictly quasi-concave ordering with quasi bliss point $x_{S}$. The collection of $2^{N}$ strictly quasi-concave preferences characterizes an Arrovian welfare function up to some tie-breaking rule. Here, the key is to show that if the ranking over two alternatives is not determined, then this does not cause any intransitivities, no matter what ranking we choose between these two alternatives. Of course, this is only possible if the two alternatives belong to opposite sides of the quasi bliss point of the social ordering and could form an indifference class.

Our characterization is one of the few positive results in Arrovian social choice. A corollary of our result is that simple majority voting is transitive if and only if individual preferences are strictly quasi-concave.

If the policy space is multi-dimensional and unbounded, and preferences are Euclidean, then Arrow's requirements are inconsistent (Border, 1984). Other proofs show that the domain of Euclidean preferences is "saturating" and apply a result due to Kalai, Muller, and Satterthwaite (1979) to deduce Arrow's impossibility (Le Breton and Weymark, 1996, 2000a,b; Campbell, 1993²). However, the proofs of these results rely importantly on the unboundedness of the policy space. As far as we know, Arrow's theorem has not been established in spatial environments when the set of alternatives is compact and convex. It is natural to assume that a govern-

\footnotetext{
${ }^{2}$ Campbell (1993) drops weak Pareto and imposes instead continuity of social preference.
} 
ment faces budget constraints and therefore the set of policy alternatives becomes bounded. We derive Arrow's theorem in such spatial environments. If individual preferences are Euclidean and the set of alternatives has a non-empty interior and it is compact and convex, then weak Pareto, independence of irrelevant alternatives, and non-dictatorship are inconsistent, i.e. again decisiveness of a coalition spreads from one pair of alternatives to all pairs of alternatives and dictatorship results.

A parallel line of research considers social choice functions. In this case, the objective is to choose for each admissible set, called an agenda, the socially optimal alternatives in this set. Arrow's choice axiom requires that if for an admissible set and some admissible subset of it, some choices made at the former set belong to the smaller set, then the choices at the latter set are exactly the choices made at the former set that belong to the smaller set (Arrow, 1959). ${ }^{3}$ In spatial environments with one-dimensional policy spaces and agenda domain consisting of all compact intervals, the social choice functions satisfying weak Pareto, Arrow's choice axiom, and independence of infeasible alternatives has been investigated (Moulin, 1984; Ehlers, 2001). These papers obtain characterizations of the "generalized median rules" by these and additional axioms. A generalized median rule assigns to each compact interval and each profile the point belonging to the compact interval that is closest to the median of the peaks reported by the voters and $2^{N}$ fixed voters. The quasi bliss points of the $2^{N}$ fixed strictly quasi-concave preferences play the role of these fixed voters. The quasi bliss point of the social ordering chosen by an Arrovian welfare function for a profile of preferences is the outcome of the generalized median rule applied to this profile of quasi bliss points. However, the natural correspondence between generalized median rules and Arrovian welfare functions fails because the median need not be the socially most preferred alternative. The class of welfare functions underlying median rules is smaller than the class of Arrovian welfare functions: in the former the social ordering is single-peaked.

The same applies to the strand of literature on strategy-proof social choice rules when the set of alternatives is one-dimensional (Moulin, 1980; Border and Jordan,

\footnotetext{
${ }^{3}$ Dutta, Jackson, and Le Breton (2001) consider Arrovian-type axioms in elections in which candidates have the possibilty to withdraw. See also Ehlers and Weyamrk (2001).
} 
1983; Kim and Roush, 1984; Barberà, Gul, and Stacchetti, 1993). For the case of a finite set of alternatives and unrestricted domain, Satterthwaite (1975) associates with each social choice rule satisfying strategy-proofness and unanimity an Arrovian welfare function. For his construction it is essential that any pair of alternatives can be moved to the top two positions in each agent's preference relation. It is obvious that this trick does not work in spatial environments.

If the policy space is at least two-dimensional, preferences are strictly quasiconcave and have bliss points, and the agenda domain consists of all compact and convex sets (including line segments), then an Arrovian choice function must be dictatorial (Duggan, 1996). However, if the agenda domain consists only of non-degenerate compact and convex sets and preferences are Euclidean, then Bergson-Samuelson welfare functions are Arrovian (Le Breton and Weymark, 2000b).

The paper is organized as follows. In Section 2 we introduce our notation and the main definitions. In Section 3 we characterize the welfare functions satisfying weak Pareto and independence of irrelevant alternatives if the policy space is onedimensional. In Section 4 we show that weak Pareto, independence of irrelevant alternatives, and non-dictatorship are inconsistent if the policy space is compact, convex, and at least two-dimensional. Section 5 concludes.

\section{Notation and Definitions}

We use the same terminology and notation as Le Breton and Weymark (2000a). Let $N \equiv\{1,2, \ldots, n\}$ denote a finite set of agents with $n \geq 2$, and let $A \subseteq \mathbb{R}^{m}$ denote a set of alternatives. Each point in $\mathbb{R}^{m}$ identifies the changes in the level of $m$ different policies, for example public spending on police, health care, and so on. Let $\mathcal{W}$ denote the set of all complete and transitive relations over $A$. An element of $\mathcal{W}$ is called a weak ordering over $A$. Given $R_{i} \in \mathcal{W}$, the corresponding strict relation, $P_{i}$, and the indifference relation, $I_{i}$, are defined as follows: for all $a, b \in A$, (i) $a P_{i} b \Leftrightarrow \neg b R_{i} a$, and (ii) $a I_{i} b \Leftrightarrow a R_{i} b$ and $b R_{i} a$. Note that if $\neg a R_{i} b$, then by completeness of $R_{i}, a R_{i} b$. Hence, $\neg b R_{i} a$ is enough to describe the strict relation $P_{i}$. Let $\mathcal{R} \subseteq \mathcal{W}$.

A (preference) profile is a list $R \equiv\left(R_{i}\right)_{i \in N} \in \mathcal{R}^{N}$. A (social) welfare function 
associates with each profile a weak ordering over $A$. Formally, a welfare function is a mapping $f: \mathcal{R}^{N} \rightarrow \mathcal{W}$ such that for all $R \in \mathcal{R}^{N}, f(R) \in \mathcal{W}$. We call $f(R)$ the social ordering (that $f$ associates with $R$ ). Note that $f(R)$ need not belong to the individual preference domain $\mathcal{R}$. Other authors impose the restriction that any social ordering belongs to each individual's preference domain (for example, Peters, van der Stel, and Storcken, 1992, and Bossert and Weymark, 1993).

Arrow's requirements are as follows. The first axiom says that if all agents strictly prefer $a$ to $b$, then $a$ should also be socially strictly preferred to $b$.

Weak Pareto: For all $R \in \mathcal{R}^{N}$ and all $a, b \in A$, if for all $i \in N, a P_{i} b$, then $\neg b f(R) a$.

Given $R \in \mathcal{R}^{N}, X \subseteq A$, and $j \in N$, let $R_{j} \mid X$ denote the restriction of $R_{j}$ to $X$, and $R \mid X \equiv\left(R_{i} \mid X\right)_{i \in N}$. The second axiom says that the social ordering of two alternatives only depends on the profile of individual preferences restricted to these two alternatives.

Independence of Irrelevant Alternatives: For all $R, \bar{R} \in \mathcal{R}^{N}$ and all $a, b \in A$, if $R|\{a, b\}=\bar{R}|\{a, b\}$, then $f(R)|\{a, b\}=f(\bar{R})|\{a, b\}$.

A welfare function is called to be Arrovian if it satisfies weak Pareto and independence of irrelevant alternatives. A welfare function is dictatorial if there exists some agent such that for each profile the social strict preference relation respects the strict preference relation of this agent.

Non-Dictatorship: There exists no $i \in N$ such that for all $R \in \mathcal{R}^{N}$ and all $a, b \in A$, if $a P_{i} b$, then $\neg b f(R) a$.

A welfare function treats individuals symmetrically if for all permutations of individuals' preferences, the social ordering remains unchanged. 
Anonymity: For all $R \in \mathcal{R}^{N}$ and all permutations $\sigma$ of $N, f(\sigma(R))=f(R)$.

\section{One-Dimensional Policy Spaces}

In this section the policy space is a one-dimensional continuum. Specifically, the set of alternatives is the set of real numbers $\mathbb{R}$. For example, in an election each element of $\mathbb{R}$ represents a candidate's political ideology on a left-right spectrum.

Given $i \in N$, a weak ordering $R_{i} \in \mathcal{W}$ is single-peaked if there exists a point $p\left(R_{i}\right) \in \mathbb{R}$, called the peak of $R_{i}$, such that for all $a, b \in \mathbb{R}$, if $a<b \leq p\left(R_{i}\right)$ or $p\left(R_{i}\right) \leq b<a$, then $b P_{i} a$. Let $\mathcal{R}$ denote the set of all single-peaked preferences over $\mathbb{R}$. Each agent $i \in N$ has a single-peaked preference relation over $\mathbb{R}$. A single-peaked preference $R_{i} \in \mathcal{R}$ is symmetric if for all $a, b \in \mathbb{R}, a R_{i} b \Leftrightarrow\left|a-p\left(R_{i}\right)\right| \leq\left|b-p\left(R_{i}\right)\right|$.

We will give a complete characterization of all welfare functions satisfying weak Pareto and independence of irrelevant alternatives.

\section{$3.1 \quad$ Decisiveness}

Given $S \subseteq N$ and $a, b \in A$, we say that $S$ is semi-decisive over " $a$ preferred to $b$ " if there exists $R \in \mathcal{R}^{N}$ such that (i) for all $i \in S, a P_{i} b$, (ii) for all $i \in N \backslash S, b P_{i} a$, and (iii) af $(R) b .{ }^{4}$ Let $D^{s}(a, b)$ denote the set of all coalitions that are semi-decisive over " $a$ preferred to $b$ ". Given $a, b \in A$ and $R \in \mathcal{R}$, if $\neg b f(R) a$ whenever $a P_{i} b$ for all $i \in S$, then $S$ is said to be decisive over " $a$ preferred to $b$ ". Let $D(a, b)$ denote the set of all coalitions that are decisive over " $a$ preferred to $b$ ".

One of the important steps in Arrow's impossibility theorem is that whenever a coalition $S \subseteq N$ is decisive over " $a$ preferred to $b$ ", then it is also decisive over " $a$ preferred to $c$ " and " $c$ preferred to $b$ " for any other alternative $c$. Therefore, every coalition is either decisive over every pair of alternatives or over no pair.

Because here individual preferences are restricted to be single-peaked and the policy space is one-dimensional, decisiveness of a coalition does not spread over all pairs

\footnotetext{
${ }^{4}$ The literature on Arrovian social choice often refers to our definition as "a coalition is semidecisive over the pair $(a, b)$ ".
} 
of alternatives as in Arrow's original theorem. Decisiveness of a coalition expands in a weaker form. Given $a, b \in \mathbb{R}$ such that $a<b$, if $S$ is semi-decisive over " $a$ preferred to $b$ ", then $S$ is decisive over " $a$ preferred to $c$ " for all $c \in] b,+\infty[$ and $S$ is decisive over " $c$ preferred to $b$ " for all $c \in] a, b[$.

Lemma 3.1 Let $f: \mathcal{R}^{N} \rightarrow \mathcal{W}$ be a welfare function satisfying weak Pareto and independence of irrelevant alternatives. Let $S \subseteq N$ and $a, b, c \in \mathbb{R}$ be such that $a<b<c$ or $a>b>c$.

(i) If $S \in D^{s}(a, b)$, then $S \in D(a, c)$.

(ii) If $S \in D^{s}(a, c)$, then $S \in D(b, c)$.

Proof. First, we show (i). Let $R \in \mathcal{R}^{N}$ be such that for all $i \in S, a P_{i} c$. We have to show that $\neg c f(R) a$. Let $\tilde{R} \in \mathcal{R}^{N}$ be such that

(a) for all $i \in N, \tilde{R}_{i}\left|\{a, c\}=R_{i}\right|\{a, c\}$;

(b) for all $i \in S, a \tilde{P}_{i} b$ and $b \tilde{P}_{i} c$; and

(c) for all $i \in N \backslash S, b \tilde{P}_{i} a$ and $b \tilde{P}_{i} c$.

It is easy to check that we can find $\tilde{R} \in \mathcal{R}^{N}$ such that (a) to (c) are satisfied (for all $i \in N$, we can even choose $\tilde{R}_{i}$ to be symmetric). By (b) and (c), for all $i \in N, b \tilde{P}_{i} c$. Thus, by weak Pareto, $\neg c f(\tilde{R}) b$. Because $S \in D^{s}(a, b)$ and $f$ satisfies independence of irrelevant alternatives, (b) and (c) imply af $(\tilde{R}) b$. Thus, by transitivity of $f(\tilde{R})$, $\neg c f(\tilde{R}) a$. Hence, by (a) and independence of irrelevant alternatives, $\neg c f(R) a$ and $S \in D(a, c)$, the desired conclusion.

Second, we show (ii). Let $R \in \mathcal{R}^{N}$ be such that for all $i \in S, b P_{i} c$. We have to show that $\neg c f(R) b$. Let $\tilde{R} \in \mathcal{R}^{N}$ be such that

(a) for all $i \in N, \tilde{R}_{i}\left|\{b, c\}=R_{i}\right|\{b, c\}$,

(b) for all $i \in S, b \tilde{P}_{i} a$ and $a \tilde{P}_{i} c$, and

(c) for all $i \in N \backslash S, b \tilde{P}_{i} a$ and $c \tilde{P}_{i} a$. 
(Again, for all $i \in N, \tilde{R}_{i}$ can be chosen to be symmetric.) By weak Pareto, $\neg a f(\tilde{R}) b$. Because $S \in D^{s}(a, c)$, we have af $(\tilde{R}) c$. Thus, by transitivity of $f(\tilde{R}), \neg c f(\tilde{R}) b$. By (a) and independence of irrelevant alternatives, $\neg c f(R) b$. Hence, $S \in D(b, c)$, the desired conclusion.

\subsection{Social Orderings at Maximal Conflicts}

In avoiding limits of profiles we add two non-single-peaked preferences to the set $\mathcal{R}$. Obviously, $\geq$ and $\leq$ are preferences over $\mathbb{R}$, where $\geq$ has a bliss point at $+\infty$ and $\leq$ at $-\infty$.

Let $\tilde{\mathcal{R}} \equiv \mathcal{R} \cup\{\geq, \leq\}$. The following lemma shows that the addition of $\geq$ and $\leq$ to $\mathcal{R}$ has no influence on a welfare function satisfying weak Pareto and independence of irrelevant alternatives.

Lemma 3.2 Let $f: \mathcal{R}^{N} \rightarrow \mathcal{W}$ be a welfare function satisfying weak Pareto and independence of irrelevant alternatives. Define $\tilde{f}: \tilde{\mathcal{R}}^{N} \rightarrow \mathcal{W}$ as follows: for all $R \in \tilde{\mathcal{R}}^{N}$ and all $a, b \in \mathbb{R}$, take $\bar{R} \in \mathcal{R}^{N}$ such that $\bar{R}|\{a, b\}=R|\{a, b\}$ and set

$$
\tilde{f}(R)|\{a, b\} \equiv f(\bar{R})|\{a, b\}
$$

Then $\tilde{f}$ is a well-defined welfare function satisfying weak Pareto and independence of irrelevant alternatives. Moreover, $\tilde{f} \mid \mathcal{R}^{N}=f$.

Proof. Let $R \in \tilde{\mathcal{R}}^{N}$. Because $f$ satisfies independence of irrelevant alternatives, for all $a, b \in \mathbb{R}, \tilde{f}(R) \mid\{a, b\}$ is well-defined. Thus, $\tilde{f}$ satisfies weak Pareto and independence of irrelevant alternatives. Obviously, $\tilde{f}(R)$ is complete. It remains to show that $\tilde{f}(R)$ is transitive. Let $a, b, c \in \mathbb{R}$ be such that $a \leq b \leq c$. Let $\bar{R} \in \mathcal{R}^{N}$ be such that for all $i \in N$, (i) if $R_{i}=\leq$, then $p\left(\bar{R}_{i}\right)=a$, (ii) if $R_{i} \in \mathcal{R}$, then $\bar{R}_{i}=R_{i}$, and (iii) if $R_{i}=\geq$, then $p\left(\bar{R}_{i}\right)=c$. By definition, $\tilde{f}(R)|\{a, b, c\}=f(\bar{R})|\{a, b, c\}$ and $\tilde{f}(R)$ is transitive.

Given a coalition $S \subseteq N$, we consider the profile where the members belonging to $S$ announce $\leq$ and the other agents announce $\geq$. Clearly, the coalitions $S$ and $N \backslash S$ 
disagree on every pair of different alternatives. We show that the social orderings at these profiles of maximal conflicts are strictly quasi-concave. They also satisfy a certain monotonicity property and determine for each profile the social ordering up to some tie-breaking when social preference can be chosen to be arbitrary.

Throughout the remaining part of Subsection 3.2, let $f$ be a welfare function satisfying weak Pareto and independence of irrelevant alternatives. For all $S \subseteq N$, let $\left(\leq_{S}, \geq_{N \backslash S}\right)$ be the profile $R \in \tilde{\mathcal{R}}^{N}$ such that for all $i \in S, R_{i}=\leq$, and for all $i \in N \backslash S$, $R_{i}=\geq$. Then for all $S \subseteq N$, let $Q^{S} \equiv \tilde{f}\left(\leq_{S}, \geq_{N \backslash S}\right)$. Let $Q \equiv\left\{Q^{S} \mid S \subseteq N\right\}$. We call $Q$ a collection of calibration relations. We will show that $Q$ completely determines $f$ (up to some tie-breaking). By Lemma 3.2, $\tilde{f}$ satisfies weak Pareto. Thus, $Q^{\emptyset}=\geq$ and $Q^{N}=\leq$. The following lemma specifies some properties of the collection $Q$.

A weak ordering $R_{i} \in \mathcal{W}$ is strictly quasi-concave, if for all $a, b, c \in \mathbb{R}$ such that $a<b<c$, we have $\neg a R_{i} b$ or $\neg c R_{i} b$. In other words, $b$ is never a worst alternative in $\{a, b, c\}$. Let $\mathcal{C}$ denote the set of all strictly quasi-concave orderings over $\mathbb{R}$.

Lemma 3.3 (i) The collection $Q$ is (inclusive) monotone, i.e. for all $S, T \subseteq N$ such that $S \subseteq T$, and all $a, b, c, d \in \mathbb{R}$ such that $a<b<c<d$, (i.i) if $b Q^{S} c$, then $\neg d Q^{T} b$; and (i.ii) if $c Q^{T} b$, then $\neg a Q^{S} c$.

(ii) For all $S \subseteq N, Q^{S} \in \mathcal{C}$.

Proof. (i) By definition, $Q^{S}=\tilde{f}\left(\leq_{S}, \geq_{N \backslash S}\right)$ and $Q^{T}=\tilde{f}\left(\leq_{T}, \geq_{N \backslash T}\right)$. If $b Q^{S} c$, then $S \in D^{s}(b, c)$. Thus, by Lemma 3.1, $S \in D(b, d)$. Hence, by $S \subseteq T$, we have $\neg d Q^{T} b$. Statement (i.ii) follows similarly.

(ii) Let $a, b, c \in \mathbb{R}$ be such that $a<b<c$. Suppose that $a Q^{S} b$ and $c Q^{S} b$. Then by (i.i), $\neg c Q^{S} a$, and by (i.ii), $\neg a Q^{S} c$, which contradicts completeness of $Q^{S}$.

Note that a strictly quasi-concave preference $R_{i} \in \mathcal{C}$ is either equal to $\leq$ or $\geq$, or there exists a quasi bliss point $p\left(R_{i}\right) \in \mathbb{R}$ (for convenience we use the same notation as for peaks) such that (i) for all $a, b \in \mathbb{R}$, if $a<b<p\left(R_{i}\right)$ or $p\left(R_{i}\right)<b<a$, then $b P_{i} a$, and (ii) either (for all $\left.x \in\right]-\infty, p\left(R_{i}\right)\left[, p\left(R_{i}\right) P_{i} x\right.$ ), or (for all $\left.x \in\right] p\left(R_{i}\right),+\infty[$, $\left.p\left(R_{i}\right) P_{i} x\right)$. We use the convention that $p(\leq) \equiv-\infty$ and $p(\geq) \equiv+\infty$. 
Obviously, $\mathcal{R} \subseteq \mathcal{C}$. However, a strictly quasi-concave preference $R_{i}$ need not be single-peaked, even if $p\left(R_{i}\right) \in \mathbb{R}$. For example, let $u: \mathbb{R} \rightarrow \mathbb{R}$ be such that for all $x \in \mathbb{R}, u(x) \equiv \frac{1}{x}$ if $x>0$ and $u(x)=x$ if $x \leq 0$. Let $R_{u} \in \mathcal{W}$ be such that for all $a, b \in \mathbb{R}, a R_{u} b \Leftrightarrow u(a) \geq u(b)$. Then $R_{u} \in \mathcal{C} \backslash \mathcal{R}$. Next, we show that for each profile the social ordering is strictly quasi-concave.

Let $R \in \mathcal{R}^{N}$ be such that $p\left(R_{i_{1}}\right) \leq p\left(R_{i_{2}}\right) \leq \cdots \leq p\left(R_{i_{n}}\right)$. Let $S_{0} \equiv \emptyset$ and for all $t \in\{1,2, \ldots, n\}, S_{t} \equiv\left\{i_{1}, i_{2}, \ldots, i_{t}\right\}$. Thus, $S_{n}=N$. The median of $M(R)=\left\{p\left(R_{i_{1}}\right), p\left(R_{i_{2}}\right), \ldots, p\left(R_{i_{n}}\right), p\left(Q^{S_{0}}\right), p\left(Q^{S_{1}}\right), \ldots, p\left(Q^{S_{n}}\right)\right\}$ is the number $m(R) \in \mathbb{R}$ such that (i) $m(R) \in M(R)$, (ii) at least $n+1$ elements of $M(R)$ are smaller than or equal to $m(R)$, and (iii) at least $n+1$ elements of $M(R)$ are greater than or equal to $m(R)$.

Theorem 3.1 For all $R \in \mathcal{R}^{N}, f(R)$ is strictly quasi-concave with quasi bliss point $m(R)$. In particular, if $m(R) \in\left\{p\left(R_{1}\right), \ldots, p\left(R_{n}\right)\right\}$, then $f(R)$ is single-peaked.

Proof. Let $R \in \mathcal{R}^{N}$. Without loss of generality, suppose $p\left(R_{1}\right) \leq p\left(R_{2}\right) \leq \cdots \leq$ $p\left(R_{n}\right)$. By independence of irrelevant alternatives, we have $\left.\left.f(R) \mid\right]-\infty, p\left(R_{1}\right)\right]=$ $\left.\left.\left.\left.Q^{\emptyset} \mid\right]-\infty, p\left(R_{1}\right)\right]=\geq \mid\right]-\infty, p\left(R_{1}\right)\right]$, for all $t \in\{1,2, \ldots n-1\}$,

$$
f(R)\left|\left[p\left(R_{t}\right), p\left(R_{t+1}\right)\right]=Q^{S_{t}}\right|\left[p\left(R_{t}\right), p\left(R_{t+1}\right)\right]
$$

and $f(R) \mid\left[p\left(R_{n}\right),+\infty\left[=Q^{N} \mid\left[p\left(R_{n}\right),+\infty\left[=\leq \mid\left[p\left(R_{n}\right),+\infty[\right.\right.\right.\right.\right.$.

Let $a, b \in \mathbb{R}$ be such that $m(R)<a<b$. We show that $\neg b f(R) a$. If $a \geq p\left(R_{n}\right)$, then the assertion follows from $f(R) \mid\left[p\left(R_{n}\right),+\infty\left[=\leq \mid\left[p\left(R_{n}\right),+\infty\left[\right.\right.\right.\right.$. Let $t^{\prime} \in$ $\{1,2, \ldots, n-1\}$ be such that $a \in\left[p\left(R_{t^{\prime}}\right), p\left(R_{t^{\prime}+1}\right)[\right.$ and $c \in] a, p\left(R_{t^{\prime}+1}\right)[\cap] a, b[$. We claim that $p\left(Q^{S_{t^{\prime}}}\right) \leq m(R)$. If $p\left(Q^{S_{t^{\prime}}}\right)>m(R)$, then at least $n+1$ numbers are greater than $m(R)$, namely $\left\{p\left(Q^{S_{0}}\right), p\left(Q^{S_{1}}\right), \ldots, p\left(Q^{S_{t^{\prime}}}\right), p\left(R_{t^{\prime}+1}\right), p\left(R_{t^{\prime}+2}\right), \ldots, p\left(R_{n}\right)\right\}$, which contradicts the median property of $m(R)$. Thus, $p\left(Q^{S_{t^{\prime}}}\right) \leq m(R)$. By (1), $f(R)\left|\left[p\left(R_{t^{\prime}}\right), p\left(R_{t^{\prime}+1}\right)\right]=Q^{S_{t^{\prime}}}\right|\left[p\left(R_{t^{\prime}}\right), p\left(R_{t^{\prime}+1}\right)\right]$. Hence, $\neg c f(R) a$. Thus, $S_{t^{\prime}} \in D^{s}(a, c)$. By Lemma 3.1 and $a<c<b, S_{t^{\prime}} \in D(a, b)$. Hence, $\neg b f(R) a$, the desired conclusion. Similarly it can be shown that for all $a, b \in \mathbb{R}$, if $b<a<m(R)$, then $\neg b f(R) a$.

Because $p\left(Q^{\emptyset}\right)=+\infty$ and $p\left(Q^{N}\right)=-\infty$, we have $m(R) \in\left[p\left(R_{1}\right), p\left(R_{n}\right)\right]$. Let $t^{\prime \prime} \in$ $\{1,2, \ldots, n-1\}$ be such that $m(R) \in\left[p\left(R_{t^{\prime \prime}}\right), p\left(R_{t^{\prime \prime}+1}\right)\right]$. If $\left.m(R) \in\right] p\left(R_{t^{\prime \prime}}\right), p\left(R_{t^{\prime \prime}+1}\right)[$, 
then $f(R) \mid] p\left(R_{t^{\prime \prime}}\right), p\left(R_{t^{\prime \prime}+1}\right)\left[=Q^{S_{t^{\prime \prime}}} \mid\right] p\left(R_{t^{\prime \prime}}\right), p\left(R_{t^{\prime \prime}+1}\right)[\operatorname{implies} p(f(R))=m(R)=$ $p\left(Q^{S_{t^{\prime \prime}}}\right)$ and $f(R) \in \mathcal{C}$. If $m(R)=p\left(R_{t^{\prime \prime}}\right)$, then similar arguments as above imply that $p\left(Q^{S_{t^{\prime \prime}}}\right) \leq m(R) \leq p\left(Q^{S_{t^{\prime \prime}-1}}\right)$. Thus, by $(1)$, it follows that $p(f(R))=m(R)=p\left(R_{t^{\prime \prime}}\right)$ and $f(R) \in \mathcal{R}$.

Remark 3.1 If for all $S \subseteq N, Q^{S} \in\{\leq, \geq\}$, then for all $R \in \mathcal{R}^{N}, f(R)$ is a singlepeaked preference relation.

By Theorem 3.1, for all $R \in \mathcal{R}^{N}$, the collection $Q$ determines the social ordering $f(R)$ restricted to $]-\infty, m(R)[$ and to $] m(R),+\infty[$ (and either on ] $-\infty, m(R)]$ or $[m(R),+\infty[)$. However, the quasi bliss point $m(R)$ needs not be the socially most preferred alternative. Next we show that $Q$ also determines the ranking of $f(R)$ over all pairs $a, b \in \mathbb{R}$ such that $a \leq m(R) \leq b$ (up to some arbitrary tie-breaking).

Lemma 3.4 Let $R \in \mathcal{R}^{N}$ and $a, b \in \mathbb{R}$ be such that $a<b$. Let $S \equiv\left\{i \in N \mid a P_{i} b\right\}$, $U \equiv\left\{i \in N \mid b P_{i} a\right\}$, and $T \equiv N \backslash(S \cup U)$. Then the following holds.

(i) If for some $x \in] a, b\left[, a Q^{S} x\right.$, or for some $\left.x \in\right]-\infty, a\left[, x Q^{S} b\right.$, then $\neg b f(R) a$.

(ii) If for some $x \in] a, b\left[, b Q^{S \cup T} x\right.$, or for some $\left.x \in\right] b,+\infty\left[, x Q^{S \cup T} a\right.$, then $\neg a f(R) b$.

(iii) If the presumptions of (i) and (ii) do not hold, then for all $x \in] a, b[$, we have $\neg a f(R) x$ and $\neg b f(R) x$, and for all $x \in]-\infty, a[\cup] b,+\infty[$, we have $\neg x f(R) a$ and $\neg x f(R) b$.

Proof. By definition, for all $i \in T, a I_{i} b$.

First, we show (i). If for some $x \in] a, b\left[, a Q^{S} x\right.$, then $S \in D^{s}(a, x)$. Thus, by Lemma 3.1 and $a<x<b, S \in D(a, b)$ and $\neg b f(R) a$. If for some $x \in]-\infty, a\left[, x Q^{S} b\right.$, then $S \in D^{s}(x, b)$. Thus, by Lemma 3.1 and $x<a<b, S \in D(a, b)$ and $\neg b f(R) a$.

Second, we show (ii). If for some $x \in] a, b\left[, b Q^{S \cup T} x\right.$, then $U \in D^{s}(b, x)$. Thus, by Lemma 3.1 and $b>x>a, U \in D(b, a)$ and $\neg a f(R) b$. If for some $x \in] b,+\infty[$, $x Q^{S \cup T} a$, then $U \in D^{s}(x, a)$. Thus, by Lemma 3.1 and $x>b>a, U \in D(b, a)$ and $\neg a f(R) b$.

Third, we show (iii). Because the presumptions of (i) and (ii) do not hold, we have 
(a) for all $x \in]-\infty, a\left[, \neg x Q^{S} b\right.$;

(b) for all $x \in] a, b\left[, \neg a Q^{S} x\right.$ and $\neg b Q^{S \cup T} x$; and

(c) for all $x \in] b,+\infty\left[, \neg x Q^{S \cup T} a\right.$.

Let $x \in]-\infty, a[$. We want to show that $\neg x f(R) a$ and $\neg x f(R) b$. Because for all $i \in N, R_{i}$ is single-peaked, we have for all $i \in T \cup U, a P_{i} x$ and $b P_{i} x$. Let $\left.y \in\right] x, a[$. By (a), $b Q^{S} y$. Thus, $T \cup U \in D^{s}(b, y)$. By Lemma 3.1 and $b>y>x, T \cup U \in D(b, x)$. Hence, $\neg x f(R) b$. By Theorem 3.1, $f(R) \in \mathcal{C}$. Thus, from $x<a<b$ and $\neg x f(R) b$ we obtain $\neg x f(R) a$.

Let $x \in] a, b[$. We want to show that $\neg a f(R) x$ and $\neg b f(R) x$. Because for all $i \in N, R_{i}$ is single-peaked, we have for all $i \in S, x P_{i} b$, for all $i \in U, x P_{i} a$, and for all $i \in T, x P_{i} a$ and $x P_{i} b$. Let $\left.y \in\right] x, b\left[\right.$. By (b), $y Q^{S} a$ and $T \cup U \in D^{s}(y, a)$. Thus, by Lemma 3.1 and $y>x>a, T \cup U \in D(x, a)$ and $\neg a f(R) x$. Let $z \in] a, x[$. By (b), $z Q^{S \cup T} b$ and $S \cup T \in D^{s}(z, b)$. Thus, by Lemma 3.1 and $z<x<b, S \cup T \in D(x, b)$ and $\neg b f(R) x$.

Let $x \in] b,+\infty[$. We want to show that $\neg x f(R) a$ and $\neg x f(R) b$. Because for all $i \in N, R_{i}$ is single-peaked, we have for all $i \in S \cup T, a P_{i} x$ and $b P_{i} x$. Let $\left.y \in\right] b, x[$. By (c), $a Q^{S \cup T} y$. Thus, $S \cup T \in D^{s}(a, y)$. By Lemma 3.1 and $a<y<x, S \cup T \in D(a, x)$. Hence, $\neg x f(R) a$. By Theorem 3.1, $f(R) \in \mathcal{C}$. Thus, from $a<b<x$ and $\neg x f(R) a$ we obtain $\neg x f(R) b$.

In (i) and (ii) of Lemma 3.4, the ranking of $f(R)$ over $\{a, b\}$ is uniquely determined by $Q$. If neither (i) nor (ii) hold, then the ranking of $f(R)$ over $\{a, b\}$ can be taken arbitrarily without causing intransitivities.

\subsection{The Characterization}

In the previous subsection we identified a monotone collection $Q$ of strictly quasiconcave orderings from a welfare function satisfying weak Pareto and independence of irrelevant alternatives. Theorem 3.1 and Lemma 3.4 imply that the welfare function 
is completely determined by $Q$. These results reveal the characteristics of such a welfare function.

In formulating the characterization result, we need a precise definition of tiebreaking. Given $a, b \in \mathbb{R}$, let $\mathcal{W}_{\{a, b\}}$ denote the set of weak orderings over $\{a, b\}$. Obviously, only three orderings over $\{a, b\}$ are possible: $a$ is strictly preferred to $b$, $a$ and $b$ are indifferent, and $b$ is strictly preferred to $a$. A tie-breaking rule $\tau$ is a family of functions $\tau_{\{a, b\}}$, indexed by $\{a, b\}$ (where $a, b \in \mathbb{R}$ and $a \neq b$ ), from $\mathcal{W}_{\{a, b\}}^{N}$ to $\mathcal{W}_{\{a, b\}}$. The function $\tau_{\{a, b\}}$ assigns to each profile of orderings over $\{a, b\}$ an element in $\mathcal{W}_{\{a, b\}}$. Tie-breaking should not be read to be strict because the resulting ordering over $\{a, b\}$ may be indifference.

Fixed-Strictly-Quasi-Concave Welfare Function, $f_{\tau}^{Q}:$ Given a monotone collection $Q$ of strictly quasi-concave orderings such that $Q^{\emptyset}=\geq$ and $Q^{N}=\leq$, and a tie-breaking rule $\tau$, the fixed-strictly-quasi-concave welfare function $f_{\tau}^{Q}$ associated with $Q$ and $\tau$ is defined as follows. Let $R \in \mathcal{R}^{N}$ and $a, b \in \mathbb{R}$ be such that $a \leq b$. Let $S \equiv\left\{i \in N \mid a P_{i} b\right\}, U \equiv\left\{i \in N \mid b P_{i} a\right\}$, and $T \equiv N \backslash(S \cup U)$. Then

$(\alpha)$ if $a=b$, then $a f_{\tau}^{Q}(R) b$;

$(\beta)$ if $a<b$ and for some $x \in] a, b\left[, a Q^{S} x\right.$, or for some $\left.x \in\right]-\infty, a\left[, x Q^{S} b\right.$, then $a f_{\tau}^{Q}(R) b$ and $\neg b f_{\tau}^{Q}(R) a ;$

$(\gamma)$ if $a<b$ and for some $x \in] a, b\left[, b Q^{S \cup T} x\right.$, or for some $\left.x \in\right] b,+\infty\left[, x Q^{S \cup T} a\right.$, then $b f_{\tau}^{Q}(R) a$ and $\neg a f_{\tau}^{Q}(R) b$; and

$(\delta)$ if $a<b$ and (a) for all $x \in]-\infty, a\left[, \neg x Q^{S} b\right.$, (b) for all $\left.x \in\right] a, b\left[, \neg a Q^{S} x\right.$ and $\neg b Q^{S \cup T} x$, and (c) for all $\left.x \in\right] b,+\infty\left[, \neg x Q^{S \cup T} a\right.$, then $f_{\tau}^{Q}(R) \mid\{a, b\} \equiv$ $\tau_{\{a, b\}}(R \mid\{a, b\})$.

Lemma 3.5 A fixed-strictly-quasi-concave welfare function is a well-defined welfare function satisfying weak Pareto and IIA.

Proof. Let $Q$ be a monotone collection of strictly quasi-concave orderings and $\tau$ be a tie-breaking rule. Let $f_{\tau}^{Q}$ be defined as above. 
First, we prove well-definedness of $f_{\tau}^{Q}$. We have to show that $(\beta)$ and $(\gamma)$ exclude each other.

Let $a, b \in \mathbb{R}$ be such that $(\beta)$ holds for $a$ and $b$. Thus, $a<b$. If for some $x \in] a, b\left[, a Q^{S} x\right.$, then by strict quasi-concavity of $Q^{S}$ and $a<x<b, \neg b Q^{S} x$. Thus, by transitivity, $\neg b Q^{S} a$. If for some $\left.x \in\right]-\infty, a\left[, x Q^{S} b\right.$, then by strict quasi-concavity of $Q^{S}$ and $x<a<b, \neg b Q^{S} a$. Hence, in both cases we have $\neg b Q^{S} a$ and $S \in D^{s}(a, b)$.

Because $S \in D^{s}(a, b)$, by Lemma 3.1, we have for all $\left.y \in\right] a, b[, S \in D(y, b)$. Thus, for all $y \in] a, b\left[, \neg b Q^{S \cup T} y\right.$. Because $S \in D^{s}(a, b)$, by Lemma 3.1, we have for all $y \in] b,+\infty[, S \in D(a, y)$. Thus, for all $y \in] b,+\infty\left[, \neg y Q^{S \cup T} a\right.$. Hence, $(\gamma)$ does not hold.

Second, we show that $f_{\tau}^{Q}$ is a welfare function. Let $R \in \mathcal{R}^{N}$. By definition, $f_{\tau}^{Q}(R)$ is complete. It remains to show that $f_{\tau}^{Q}(R)$ is transitive. Let $a, b, c \in \mathbb{R}$ be such that $a<b<c$. If $\neg a f_{\tau}^{Q}(R) b$ and $\neg c f_{\tau}^{Q}(R) b$, then $f_{\tau}^{Q}(R) \mid\{a, b, c\}$ is transitive. Thus, in proving transitivity of $f_{\tau}^{Q}(R)$, it suffices to show the following two implications: if $a f_{\tau}^{Q}(R) b$, then $\neg c f_{\tau}^{Q}(R) a$; and if $c f_{\tau}^{Q}(R) b$, then $\neg a f_{\tau}^{Q}(R) c$.

We only prove the first implication. The second implication follows similarly. Let $a f_{\tau}^{Q}(R) b$. Then $(\beta)$ or $(\delta)$ holds for $a$ and $b$. Let $S \equiv\left\{i \in N \mid a P_{i} b\right\}, U \equiv\{i \in$ $\left.N \mid b P_{i} a\right\}, T \equiv N \backslash(S \cup U)$, and $S^{\prime} \equiv\left\{i \in N \mid a P_{i} c\right\}$. Because $a<b<c$ and for all $i \in N, R_{i}$ is single-peaked, we have $S \cup T \subseteq S^{\prime}$. We distinguish two cases.

Case 1: $a f_{\tau}^{Q}(R) b$ because of $(\beta)$.

If for some $x \in] a, b\left[, a Q^{S} x\right.$, then, because $Q$ is monotone and $S \subseteq S^{\prime}, a Q^{S^{\prime}} x$. Since $a<b<c$, we have $x \in] a, c\left[\right.$ and by $(\beta), \neg c f_{\tau}^{Q}(R) a$. If for some $\left.x \in\right]-\infty, a[$, $x Q^{S} b$, then because $Q$ is monotone and $S \subseteq S^{\prime}, x Q^{S^{\prime}} b$. Thus, by strict quasi-concavity of $Q^{S^{\prime}}$ and $x<a<b<c, x Q^{S^{\prime}} c$. Hence, by $(\beta), \neg c f_{\tau}^{Q}(R) a$.

Case 2: $a f_{\tau}^{Q}(R) b$ because of $(\delta)$.

We show that $(\beta)$ holds for $a$ and $c$. Let $x \in] b, c\left[\right.$. Then by $(\delta)$, part (c), $\neg x Q^{S \cup T} a$. Thus, $a Q^{S \cup T} x$. Because $Q$ is monotone and $S \cup T \subseteq S^{\prime}, a Q^{S^{\prime}} x$. Thus, by $\left.x \in\right] a, c[$ and $(\beta), \neg c f_{\tau}^{Q}(R) a$, the desired conclusion.

Third, we show that $f_{\tau}^{Q}$ satisfies weak Pareto and independence of irrelevant alternatives. Because $Q^{\emptyset}=\geq$ and $Q^{N}=\leq$, it is easy to check that $f_{\tau}^{Q}$ satisfies 
weak Pareto. Obviously, by definition, $f_{\tau}^{Q}$ satisfies independence of irrelevant alternatives.

The following theorem characterizes the class of welfare functions satisfying Arrow's requirements on the domain of all single-peaked preferences over a one-dimensional policy space.

Theorem 3.2 The class of fixed-strictly-quasi-concave welfare functions are characterized by weak Pareto and independence of irrelevant alternatives.

Proof. From Lemma 3.5 it follows that a fixed-strictly-quasi-concave welfare function is a well-defined welfare function satisfying weak Pareto and independence of irrelevant alternatives.

Conversely, let $f$ be a welfare function satisfying weak Pareto and independence of irrelevant alternatives. Lemma 3.3 shows the existence of a monotone collection $Q$ of strictly quasi-concave orderings. Let $R \in \mathcal{R}^{N}$ and $a, b \in \mathbb{R}$. From Lemma 3.4 it follows that $f(R) \mid\{a, b\}$ satisfies the definitions $(\alpha),(\beta)$, and $(\gamma)$. Finally, if $(\delta)$ holds, then define $\tau_{\{a, b\}}(R \mid\{a, b\}) \equiv f(R) \mid\{a, b\}$. Now it is obvious that $f$ is a fixedstrictly-quasi-concave welfare function associated with $Q$ and $\tau$.

Theorem 3.2 remains valid if the set of alternatives is a compact interval and/or each individual's preference domain is the set of all symmetric single-peaked preferences.

A social choice function aggregates profiles of individual preferences into choice functions for society. Such a choice function chooses for each permissible subset of the set of alternatives, called an agenda, the socially most preferred alternatives that belong to the agenda. If the agenda domain contains all pairs and triples of alternatives, then each social choice function satisfying weak Pareto and independence of infeasible alternatives corresponds to a social welfare function satisfying weak Pareto and independence of irrelevant alternatives (Arrow, 1959). In our model, if the agenda domain is the set of all compact intervals, then it is not possible to associate with each social welfare function a social choice function that chooses for each profile and each agenda 
the most preferred alternatives. Social preferences are only strictly quasi-concave and maxima do not necessarily exist. However, we could define a choice function that associates with each profile and each interval the quasi bliss point of the social ordering over this interval. This choice function then satisfies weak Pareto, Arrow's choice axiom, and independence of infeasible alternatives (Moulin, 1984).

\subsection{Majority Voting}

In this subsection we explore the relation between fixed-strictly-quasi-concave welfare functions and majority voting. Under majority voting, for a given profile of preferences, one alternative is ranked above another if and only if a majority of voters weakly prefers the former alternative to the latter. If the number of individuals is odd, majority voting is a well-defined welfare function (Black, 1948; Arrow, 1951). However, if the number of individuals is even, then the social indifference relation may be intransitive. In resolving this intransitivity, we add an odd number of fixed strictly quasi-concave relations and determine the majority preference relation for the profile of individual's preferences and the fixed voter's preferences.

Let $O \equiv\left(O_{0}, O_{1}, \ldots, O_{n}\right)$ denote a profile of $n+1$ fixed voters' preferences such that $O_{0}=\geq, O_{n}=\leq$, and for all $t \in\{1, \ldots, n-1\}, O_{t} \in \mathcal{C}$. Let $Q(O)$ denote the collection of strictly quasi-concave preferences associated with $O$, i.e. for all $S \subseteq N$, $Q^{S}=O_{|S|}$. We call $O$ monotone if $Q(O)$ is monotone. Given a monotone profile $O$ of $n+1$ fixed voters, the majority welfare function $g^{O}$ associated with $O$ is defined as follows. For all $R \in \mathcal{R}^{N}$ and all $a, b \in \mathbb{R}$,

$$
\begin{aligned}
& a g^{O}(R) b \Leftrightarrow\left|\left\{i \in N \mid \neg b R_{i} a\right\}\right| \cup\left|\left\{t \in\{0,1, \ldots, n\} \mid \neg b O_{t} a\right\}\right| \\
& \geq\left|\left\{i \in N \mid \neg a R_{i} b\right\}\right| \cup\left|\left\{t \in\{0,1, \ldots, n\} \mid \neg a O_{t} b\right\}\right| .
\end{aligned}
$$

The majority tie breaking rule $\tau^{O}$ associated with $O$ is defined as follows: for all $R \in \mathcal{R}^{N}$ and all $a, b \in \mathbb{R}, \tau_{\{a, b\}}^{O}(R \mid\{a, b\}) \equiv g^{O}(R) \mid\{a, b\}$.

If $O$ is a monotone profile of $n+1$ fixed voters, then the majority welfare function $g^{O}$ is the fixed-strictly-quasi-concave welfare function associated with $Q(O)$ and the majority tie-breaking rule $\tau^{O}$. 
Proposition 3.1 If $O$ is a monotone profile of $n+1$ fixed voters, then $g^{O}=f_{\tau^{O}}^{Q(O)}$.

Proof. Let $R \in \mathcal{R}^{N}$ and $a, b \in \mathbb{R}$ be such that $a<b$. Let $S \equiv\left\{i \in N \mid a P_{i} b\right\}$, $U \equiv\left\{i \in N \mid b P_{i} a\right\}$, and $T \equiv N \backslash(S \cup U)$. It suffices to show that $f_{\tau^{O}}^{Q(O)} \mid\{a, b\}=$ $g^{O}(R) \mid\{a, b\}$.

Let $a f_{\tau^{O}}^{Q(O)}(R) b$. If $a f_{\tau^{O}}^{Q(O)}(R) b$ because of $(\delta)$, then by definition of $\tau^{O}, a g^{O}(R) b$. If $a f_{\tau^{O}}^{Q(O)}(R) b$ because of $(\beta)$, then $\neg b O_{|S|} a$ and at least $n+1$ voters strictly prefer $a$ to $b$, namely $S \cup\{|S|,|S|+1, \ldots,|N|\}$. Thus, $\neg b g^{O}(R) a$. Similarly it can be shown that $b f_{\tau^{O}}^{Q(O)}(R) a$ implies $b g^{O}(R) a$.

Let $a g^{O}(R) b$. First, we show that if $\neg b O_{|S|} a$, then $(\gamma)$ does not hold. Because $O$ is monotone, we have $\neg b O_{|S \cup T|} a$. Thus, by strict quasi-concavity of $O_{|S \cup T|}$, for all $x \in] a, b\left[, \neg b O_{|S \cup T|} x\right.$, and for all $\left.x \in\right] b,+\infty\left[, \neg x O_{|S \cup T|} a\right.$, and $(\gamma)$ does not hold. Thus, if $\neg b O_{|S|} a$, then $(\beta)$ or $(\delta)$ holds for $a$ and $b$ and by definition, $a f_{\tau^{O}}^{Q(O)}(R) b$.

Similarly it can be shown if $b g^{O}(R) a$ and $\neg a O_{|S \cup T|} b$, then $(\beta)$ does not hold and $b f_{\tau^{O}}^{Q(O)}(R) a$.

If $b O_{|S|} a$ and $a O_{|S \cup T|} b$, then $(\beta)$ and $(\gamma)$ do not hold and by $(\delta), f_{\tau^{O}}^{Q(O)} \mid\{a, b\}=$ $g^{O}(R) \mid\{a, b\}$, the desired conclusion.

If $f_{\tau}^{Q}$ is an anonymous welfare function, then for all coalitions $S, Q^{S}$ depends only on the cardinality of $|S|$. In particular, the profile $\left(Q_{\emptyset}, Q_{\{1\}}, Q_{\{1,2\}}, \ldots, Q_{N}\right)$ is a profile of $n+1$ fixed voters. However, majority welfare functions are not characterized by weak Pareto, independence of irrelevant alternatives, and anonymity. This is due to the fact that the tie breaking rule $\tau$ need not be the majority tie breaking rule associated with the profile of $n+1$ fixed voters.

If $n$ is odd, for all $k \in\left\{0,1, \ldots, \frac{1}{2}(n-1)\right\}, O_{k}=\geq$, and for all $l \in\left\{\frac{1}{2}(n-1)+\right.$ $1, \ldots, n\}, O_{l}=\leq$, then Theorem 3.1 and Proposition 3.1 yield Black's celebrated median voter theorem saying that the median of the individual peaks is equal to the alternative that is top-ranked according to simple majority preference. Let $g^{m}$ denote the simple majority welfare function. As an application of our results we show the following. If the number of voters is odd and the policy space is one-dimensional, then simple majority voting is transitive if and only if each voter's preference relation 
is strictly quasi-concave.

Theorem 3.3 Let $|N|$ be odd and $|N| \neq 1$. The domain of strictly quasi-concave preferences is the unique maximal domain $\overline{\mathcal{R}}^{N}$ such that

(i) $\mathcal{R}^{N} \subseteq \overline{\mathcal{R}}^{N} \subseteq \mathcal{W}^{N}$ and

(ii) simple majority voting is transitive on the domain $\overline{\mathcal{R}}^{N}$.

Proof. Obviously, $|N| \geq 3$. It is straightforward to adjust the definition of a fixedstrictly-quasi-concave welfare function to $\mathcal{C}^{N}$ and to show that Lemma 3.5 remains true. Hence, by Proposition 3.1, simple majority voting is transitive on the domain $\mathcal{C}^{N}$

Suppose that $\overline{\mathcal{R}}^{N}$ is a domain such that $\mathcal{R}^{N} \subseteq \overline{\mathcal{R}}^{N}$ and majority voting is transitive. Thus, $g^{m}$ is a welfare function with domain $\overline{\mathcal{R}}^{N}$ satisfying weak Pareto and independence of irrelevant alternatives. Suppose that $\overline{\mathcal{R}} \backslash \mathcal{C} \neq \emptyset$. Let $R_{0} \in \overline{\mathcal{R}} \backslash \mathcal{C}$. Define $f: \mathcal{R}^{N \backslash\{n\}} \rightarrow \mathcal{W}$ as follows: for all $R \in \mathcal{R}^{N \backslash\{n\}}, f(R) \equiv g^{m}\left(R, R_{0}\right)$. Because $g^{m}$ is simple majority voting and $|N| \geq 3, f$ satisfies weak Pareto. Thus, $f$ is a welfare function satisfying weak Pareto and independence of irrelevant alternatives. Let $S \equiv\left\{1, \ldots, \frac{1}{2}(|N|-1)\right\}$. By definition of $f$ and the fact that $g^{m}$ is simple majority voting, we have $\tilde{f}\left(\leq_{S}, \geq_{N \backslash(S \cup\{n\})}\right)=R_{0}$. Because $R_{0}$ is not strictly quasi-concave, this contradicts Lemma 3.3.

\section{Multi-Dimensional Policy Spaces}

This section considers environments where the set of alternatives is multi-dimensional and connected. Let $A \subseteq \mathbb{R}^{m}$ be a set of alternatives such that the relative interior of $A$ in $\mathbb{R}^{m}$ is non-empty. A preference relation $R_{i} \in \mathcal{W}$ is Euclidean if there exists a bliss point $p\left(R_{i}\right) \in A$ such that for all $a, b \in A, a R_{i} b \Leftrightarrow\left\|a-p\left(R_{i}\right)\right\| \leq\left\|b-p\left(R_{i}\right)\right\|$ (here $\|\cdot\|$ denotes the Euclidean norm in $\mathbb{R}^{m}$ ). In this section, $\mathcal{R}$ denotes the set of all Euclidean preferences over $A$.

For the special case of $A=\mathbb{R}^{m}$, Border (1984) and Le Breton and Weymark (1996) establish Arrow's theorem. For $A=\mathbb{R}_{+}^{m}$, Le Breton and Weymark (2000a,b) show 
Arrow's theorem. However, all these results rely importantly on the unboundedness of the set of alternatives. In real life, policy makers face budget constraints and these results do not apply. Among others we show Arrow's theorem for compact and convex sets of policy alternatives.

We establish the following theorem: Arrow's requirements are inconsistent if the set of alternatives has a non-empty and connected interior and it is contained in the closure of its interior (i.e. its boundary has no "tails").

Let int $A$ denote the interior of $A$ in $\mathbb{R}^{m}$ and $\operatorname{cl}(A)$ the closure of $A$ in $\mathbb{R}^{m}$.

Theorem 4.1 Let $A \subseteq \mathbb{R}^{m}$ be such that int $A \neq \emptyset$, int $A$ is connected, and $A \subseteq$ cl(intA). If $m \geq 2$, then weak Pareto, independence of irrelevant alternatives, and non-dictatorship are inconsistent.

If the interior of the set of alternatives is non-empty and not connected, then an Arrovian social welfare function need not be dictatorial.

Example 4.1 Let $m \equiv 2, A_{1} \equiv[0,1] \times[0,1], A_{2} \equiv[0,1] \times[9,10]$, and $A \equiv A_{1} \cup A_{2}$. For all $R \in \mathcal{R}^{N}$, let $\varphi(R)\left|A_{1}=R_{1}\right| A_{1}, \varphi(R)\left|A_{2}=R_{2}\right| A_{2}$, and for all $a \in A_{1}$ and all $b \in A_{2}, \varphi(R)\left|\{a, b\}=R_{1}\right|\{a, b\}$. Then $\varphi$ is an Arrovian welfare function satisfying non-dictatorship.

The conclusion of Example 4.1 does not remain true if the sets $A_{1}$ and $A_{2}$ are connected through a line segment, for example if $A=A_{1} \cup A_{2} \cup[(0,0),(0,10)]$. For this set of alternatives an Arrovian welfare function must be dictatorial. ${ }^{5}$

If the boundary of $A$ contains tails, then again an Arrovian welfare function must not be dictatorial.

Example 4.2 Let $m \equiv 2, N \equiv\{1,2\}, B \equiv[0,1] \times[0,1]$, and $L \equiv](0,1),(0,2)]$. Let $A \equiv B \cup L$. For all $R \in \mathcal{R}^{N}$ and all $a, b \in A$, (i) if $a, b \in L$ and for some $i \in N, a R_{i} b$, then $a f(R) b$, and (ii) otherwise, $f(R)\left|\{a, b\}=R_{1}\right|\{a, b\}$. Note that for all $R \in \mathcal{R}^{N}$, if $p\left(R_{1}\right) \in B$, then $f(R)=R_{1}$. It is easy to check that $f$ is a welfare function satisfying weak Pareto, independence of irrelevant alternatives, and non-dictatorship. $\quad \triangleleft$

\footnotetext{
${ }^{5}$ By Theorem 4.1, $f \mid A_{1}$ and $f \mid A_{2}$ are dictatorial. Because $A_{1}$ and $A_{2}$ are connected through the line segment $[(0,0),(0,10)]$ it follows that the dictator of $f \mid A_{1}$ and $f \mid A_{2}$ is the same and $f$ is dictatorial.
} 


\subsection{Proof of Theorem 4.1}

Let $^{6} f$ be a welfare function satisfying weak Pareto and independence of irrelevant alternatives. Given $X \subseteq A$, let $f^{X}$ denote the restriction of $f$ to the set of alternatives $X$. Formally, for all $R \in \mathcal{R}^{N}$ such that for all $i \in N, p\left(R_{i}\right) \in X$, let $f^{X}(R \mid X) \equiv$ $f(R) \mid X$. It is straightforward that $f^{X}$ inherits weak Pareto and independence of irrelevant alternatives from $f$.

We introduce additional notation. Let $a, b, c \in \mathbb{R}^{m}$ be three distinct alternatives. Let $[a, b]$ denote the line segment between $a$ and $b$, i.e. $[a, b] \equiv\{\lambda a+(1-\lambda) b \mid \lambda \in$ $[0,1]\}$. Let $H(a, b)$ denote the hyperplane that bisects $[a, b]$ perpendicularly. In the non-degenerate case, that is $a, b$, and $c$ are non-colinear, the hyperplanes $H(a, b)$, $H(a, c)$, and $H(b, c)$ determine six disjoint open cones, say $\angle(a b c), \angle(a c b), \angle(b a c)$, $\angle(b c a), \angle(c a b)$, and $\angle(c b a)$ (see Figure 1$)$. We named these cones such that for all $R_{i} \in \mathcal{R}, p\left(R_{i}\right) \in \angle(a c b) \Leftrightarrow a P_{i} c P_{i} b$. The same holds for the other cones. Note that the collective boundary of $\angle(a b c)$ and $\angle(a c b)$ is a subset of $H(b, c)$. Let $\angle(a(b c))$ denote the set of all points $x \in H(b, c)$ such that for $R_{i} \in \mathcal{R}$, if $p\left(R_{i}\right)=x$, then $a P_{i} b I_{i} c$.

Given $a \in \mathbb{R}^{m}$ and $\epsilon>0$, let $\bigodot(a, \epsilon)$ denote the open ball with center $a$ and radius $\epsilon$, i.e. $\bigodot(a, \epsilon) \equiv\left\{x \in \mathbb{R}^{m} \mid\|a-x\|<\epsilon\right\}$. Given $a, b, c \in \mathbb{R}^{m}$, let plane $(a, b, c)$ denote the plane spanned by $a, b$, and $c$, i.e. plane $(a, b, c) \equiv\{a+\lambda(b-a)+\mu(c-a) \mid \lambda, \mu \in$ $\mathbb{R}\}$. Finally, let $\triangle(a, b, c)$ denote the triangle with corner points $a, b$, and $c$, i.e. $\triangle(a, b, c) \equiv\{\lambda a+\mu b+(1-\lambda-\mu) c \mid \lambda, \mu \geq 0 \& \lambda+\mu \leq 1\}$. The baricenter of $\triangle(a, b, c)$ is the point $\frac{1}{3}(a+b+c)$.

The following lemma is a generalizes Lemma 3.1.

Lemma 4.1 Let $S \subseteq N$ and $a, b \in A$ be such that $a \neq b$. Let $c \in A \backslash\{a, b\}$ be such that $\angle(a b c) \cap A \neq \emptyset, \angle(b a c) \cap A \neq \emptyset, \angle(b(a c)) \cap A \neq \emptyset$, and $\angle(b c a) \cap A \neq \emptyset$. If $S \in D^{s}(a, b)$, then $S \in D(a, c)$.

\footnotetext{
${ }^{6}$ The first version of the paper used Theorem 3.2 to prove Theorem 4.1 for the case when $A$ is compact and convex. A proof using Arrow's theorem was suggested by John Duggan to the first author while he was visiting University of Rochester. Independently the second author established the proof below.
} 
Proof. Let $R \in \mathcal{R}^{N}$ be such that for all $i \in S, a P_{i} c$. Let $S^{\prime} \equiv\left\{i \in N \mid a P_{i} c\right\}$, $T \equiv\left\{i \in N \mid a I_{i} c\right\}$, and $V \equiv\left\{i \in N \mid c P_{i} a\right\}$. Because $\angle(a b c), \angle(b a c), \angle(b(a c))$, and $\angle(b c a)$ have a non-empty intersection with $A$, there exists $\tilde{R} \in \mathcal{R}^{N}$ such that for all $i \in S, a \tilde{P}_{i} b \tilde{P}_{i} c$, for all $i \in S^{\prime} \backslash S, b \tilde{P}_{i} a \tilde{P}_{i} c$, for all $i \in T, b \tilde{P}_{i} a \tilde{I}_{i} c$, and for all $i \in V$, $b \tilde{P}_{i} c \tilde{P}_{i} a$. By $S \in D^{s}(a, b)$ and independence of irrelevant alternatives, $a f(\tilde{R}) b$. By weak Pareto, $\neg c f(\tilde{R}) b$. Thus, by transitivity of $f(\tilde{R}), \neg c f(\tilde{R}) a$. Hence, by independence of irrelevant alternatives, $\neg c f(R) a$ and $S \in D(a, c)$, the desired conclusion.

Note that if $A$ is the real line, then the presumptions of Lemma 4.1 only hold if $a<b<c$ or $a>b>c$. While in the one-dimensional case, Lemma 4.1 implies only a limited decisiveness power, in the multi-dimensional case decisiveness appears to be unbounded.

For the moment, suppose that $A$ is convex. Let $a, b, c \in A$ be three distinct alternatives. Then $\angle(a b c) \cap A \neq \emptyset, \angle(b a c) \cap A \neq \emptyset, \angle(b(a c)) \cap A \neq \emptyset$, and $\angle(b c a) \cap A \neq$ $\emptyset$ whenever $\angle(a b c) \cap A \neq \emptyset$ and $\angle(b c a) \cap A \neq \emptyset$. Because $A$ is convex $\angle(a b c) \cap A \neq \emptyset$ if the baricenter of $\triangle(a, b, c)$ belongs to the same half plane to which $c$ belongs induced by the line containing $[a, b]$ in plane $(a, b, c)$. This means that $\triangle(a, b, c)$ is acute at $a$. Similarly $\angle(b c a) \cap A \neq \emptyset$ if $\triangle(a, b, c)$ is acute at $c$. This is accomplished if $c$ belongs to $Y(a, b) \equiv\left\{x \in A \backslash \bigodot\left(\frac{1}{2}(a+b), \frac{1}{2}\|a-b\|\right) \mid x\right.$ is in the same open halfspace as $b$, where this halfspace is induced by the hyperplane through $a$ perpendicular to $[a, b]\}$ (see Figure 2).

These considerations together with Lemma 4.1 yield the following.

Lemma 4.2 Let $A$ be convex and $a, b \in A$ be such that $a \neq b$. If $S \in D^{s}(a, b)$, then for all $c \in Y(a, b), S \in D(a, c)$.

The following lemma expands this decisiveness power even further if $\bigodot(a,\|a-b\|)$ is a subset of $A$. Given a set $X \subseteq \mathbb{R}^{m}$, let $\partial X \equiv \operatorname{cl}(X) \backslash \operatorname{int} X$ denote the boundary of $X$.

Lemma 4.3 Let $A$ be convex and $a, b \in A$ be such that $a \neq b$ and $\odot(a,\|a-b\|) \subseteq A$. If $S \in D^{s}(a, b)$, then for all $c \in A \backslash\{a\}, S \in D(a, c)$. 
Proof. Note that by $\odot(a,\|a-b\|) \subseteq A, \cup_{x \in \partial} \odot(a,\|a-b\|) Y(a, x)=A \backslash\{a\}$. By Lemma 4.2, for all $c \in Y(a, b) \cap \partial \odot(a,\|a-b\|), S \in D(a, c)$. Because $S \in D(a, c)$ implies $S \in D^{s}(a, c)$, Lemma 4.2 implies that for all $c \in Y(a, b) \cap \partial \odot(a,\|a-b\|)$ and all $c^{\prime} \in Y(a, c), S \in D\left(a, c^{\prime}\right)$. Hence, for all $c \in A \backslash\{a\}, S \in D(a, c)$.

Next by applying Lemma 4.3 and Arrow's theorem we show that if the set of alternatives is an open ball, then $f$ is dictatorial.

Lemma 4.4 Let $x \in \mathbb{R}^{m}, \epsilon>0$, and $A=\bigodot(x, \epsilon)$. Then $f$ is dictatorial.

Proof. Let $a, b \in \odot(x, \epsilon)$ be such that $a \neq b$. Because $a, b \notin \partial \bigodot(x, \epsilon)$, there exists $c \in \odot(x, \epsilon)$ such that $\triangle(a, b, c)$ is acute. But then the baricenter of $\triangle(a, b, c)$ belongs to $\triangle(a, b, c)$. Hence, for all $u, v, w \in\{a, b, c\}$ such that $\{u, v, w\}=\{a, b, c\}, \angle(u v w) \cap$ $\odot(x, \epsilon) \neq \emptyset .^{7}$ Applying Arrow's theorem yields that $f^{\{a, b, c\}}$ is dictatorial with dictator $i_{\{a, b, c\}}$. Since $c \in \bigodot(x, \epsilon)$, there exists $\epsilon^{\prime}>0$ such that $\bigodot\left(c, \epsilon^{\prime}\right) \subseteq \bigodot(x, \epsilon)$. Now we can find $a^{\prime}, b^{\prime} \in \odot\left(c, \epsilon^{\prime}\right)$ such that $\triangle\left(a^{\prime}, b^{\prime}, c\right)$ is acute and $\odot\left(c,\left\|a^{\prime}-c\right\|\right) \subseteq$ $\bigodot\left(c, \epsilon^{\prime}\right)$. Similarly to $\triangle(a, b, c)$, Arrow's theorem implies that $f^{\left\{a^{\prime}, b^{\prime}, c\right\}}$ is dictatorial with dictator $i_{\left\{a^{\prime}, b^{\prime}, c\right\}}$. Because $\left\{i_{\left\{a^{\prime}, b^{\prime}, c\right\}}\right\} \in D\left(c, a^{\prime}\right)$ and $\odot\left(c,\left\|a^{\prime}-c\right\|\right) \subseteq \bigodot(x, \epsilon)$, Lemma 4.3 implies that for all $y \in \bigodot(x, \epsilon) \backslash\{c\},\left\{i_{\left\{a^{\prime}, b^{\prime}, c\right\}}\right\} \in D(c, y)$. Therefore, $\left\{i_{\left\{a^{\prime}, b^{\prime}, c\right\}}\right\} \in D(c, a)$ and $\left\{i_{\{a, b, c\}}\right\} \in D(c, a)$. Thus, $i_{\left\{a^{\prime}, b^{\prime}, c\right\}}=i_{\{a, b, c\}}$.

Hence, we have shown that for all $a, b \in \odot(x, \epsilon)$ such that $a \neq b$, there exists $c \in \odot(x, \epsilon)$ and $i_{\{a, b, c\}} \in N$ such that $i_{\{a, b, c\}}$ is decisive over " $a$ preferred to $b$ ", over " $b$ preferred to $a$ ", over "c preferred to $a$ ", and for all $y \in \odot(x, \epsilon) \backslash\{c\}$, over "c preferred to $y$ ". Clearly it follows that $i_{\{a, b, c\}}$ is the same individual for all those $a, b, c \in \bigodot(x, \epsilon)$. Let $i$ denote this individual. Thus, for all $a, b \in \bigodot(x, \epsilon)$ such that $a \neq b, i$ is decisive over " $a$ preferred to $b$ ". Hence, $f$ is dictatorial.

Next we show that $f^{\text {int } A}$ is dictatorial if $\operatorname{int} A$ is connected.

Lemma 4.5 Let intA be non-empty and connected. Then $f^{\text {int } A}$ is dictatorial.

\footnotetext{
${ }^{7}$ In other words, the set $\{a, b, c\}$ is a free triple.
} 
Proof. First, note that for all $x \in \operatorname{int} A$ there is some $\epsilon_{x}>0$ such that $\odot\left(x, \epsilon_{x}\right) \subseteq$ $\operatorname{int} A$. Hence, by Lemma 4.4, $\odot^{\left(x, \epsilon_{x}\right)}$ is dictatorial, say with dictator $i_{x}$.

Let $a, b \in \operatorname{int} A$ be such that $a \neq b$. It is sufficient to show that $i_{a}=i_{b}$ and $i_{a}$ is decisive over " $a$ preferred to $b$ ".

Because $\operatorname{int} A$ is connected, there is a path in $\operatorname{int} A$ from $a$ to $b$. Clearly, the path is compact and therefore, there exists a sequence $c_{0}, c_{1}, \ldots, c_{k} \operatorname{in} \operatorname{int} A$ and $\epsilon>0$ such that (i) $c_{0}=a$ and $c_{k}=b$, (ii) for all $t \in\{0,1, \ldots, k\}, \bigodot\left(c_{t}, \epsilon\right) \subseteq \operatorname{int} A$, (iii) for all $t \in\{1, \ldots, k\}, \bigodot\left(c_{t-1}, \epsilon\right) \cap\left\{c_{t}, c_{t+1}, \ldots, c_{k}\right\}=\left\{c_{t}\right\}$ and $\left\|c_{t-1}-c_{t}\right\|<\epsilon$. For all $t \in\{0,1, \ldots, k\}$, let $i_{t}$ denote the dictator of $f \odot\left(c_{t}, \epsilon\right)$.

By (i), (ii), and (iii), it follows that $i_{a}=i_{0}=i_{1}=\cdots=i_{k}=i_{b}$. Thus, $i_{a}=i_{b}$. For all $t \in\{1, \ldots, k\}$, let $R_{i_{a}}^{t} \in \mathcal{R}$ be such that $p\left(R_{i_{a}}^{t}\right)=c_{t-1}$. Thus, by (iii), $c_{t-1} P_{i_{a}}^{t} c_{t} P_{i_{a}}^{t} b$. Using these preferences, the fact that $i_{a}=i_{k-1}=i_{b}$, (iii), and transitivity, it follows that $i_{a}$ is decisive over " $c_{k-2}$ preferred to $b$ ". Then, by $i_{a}=i_{k-2}=i_{b}$ and the previous fact, $i_{a}$ is decisive over " $c_{k-3}$ preferred to $b$ ". Continuing in this way it follows that for all $t \in\{1, \ldots, k\}, i_{a}$ is decisive over " $c_{k-t}$ preferred to $b$ ". Hence, $i_{a}$ is decisive over " $a$ preferred to $b$ ", the desired conclusion.

Our final lemma completes the proof of Theorem 4.1. If $A \backslash \operatorname{int} A$ contains no "tails", i.e. $A \subseteq \operatorname{cl}(\operatorname{int} A)$, then dictatorship on the interior of $A$ expands to the whole set $A$.

Lemma 4.6 Let intA be non-empty and connected, and $A \subseteq \operatorname{cl}($ int $A)$. If $f^{\text {int } A}$ is dictatorial, then $f$ is dictatorial.

Proof. Without loss of generality, let 1 be the dictator of $f^{\text {int } A}$. We show that 1 is the dictator of $f$.

Let $a, b \in A$ be such that $a \neq b$. Let $R \in \mathcal{R}^{N}$ be such that $a P_{1} b$. It suffices to show that $\neg b f(R) a$.

Let $S \equiv\left\{i \in N \mid a P_{i} b\right\}, T \equiv\left\{i \in N \mid a I_{i} b\right\}$, and $V \equiv\left\{i \in N \mid b P_{i} a\right\}$. Because $A \subseteq \operatorname{cl}(\operatorname{int} A)$ and $\operatorname{int} A$ is connected, there exists a path from $a$ to $b \operatorname{in} \operatorname{int} A$ such that $a$ and $b$ are the only possible points on this path belonging to $A \backslash \operatorname{int} A$. Thus, $\operatorname{int} A \cap H(a, b) \neq \emptyset$. Let $h \in \operatorname{int} A \cap H(a, b)$ and $R^{\prime} \in \mathcal{R}^{N}$ be such that for all 
$i \in S, p\left(R_{i}^{\prime}\right)=a$, for all $i \in T, p\left(R_{i}^{\prime}\right)=h$, and for all $i \in V, p\left(R_{i}^{\prime}\right)=b$. Thus, by $R^{\prime}|\{a, b\}=R|\{a, b\}$ and independence of irrelevant alternatives,

$$
f(R)\left|\{a, b\}=f\left(R^{\prime}\right)\right|\{a, b\}
$$

We consider four cases.

Case 1: $a, b \in \operatorname{int} A$.

Then for all $i \in N, p\left(R_{i}^{\prime}\right) \in \operatorname{int} A$. Because $f^{i n t A}$ is dictatorial with dictator 1 and $1 \in S$, it follows that $\neg b f\left(R^{\prime}\right) a$. Hence, by $(2), \neg b f(R) a$, the desired conclusion.

Case 2: $a \in \operatorname{int} A$ and $b \in A \backslash \operatorname{int} A$.

Because $a \in \operatorname{int} A$, for some $0<\epsilon<\frac{1}{4}\|a-b\|$ we have $\odot(a, \epsilon) \subseteq \operatorname{int} A$. Let $c \in \bigodot(a, \epsilon) \cap$ plane $(a, b, h) \cap \partial \bigodot\left(\frac{1}{2}(a+b), \frac{1}{2}\|a-b\|\right)$ be such that $\|h-c\|<\| h-$ $a \|$. Because $h \in \operatorname{int} A \cap H(a, b)$ and by our choice of $c, h \in \partial \angle(c(b a))$. Thus, $\angle(c b a) \cap \operatorname{int} A \neq \emptyset$. Let $R^{\prime \prime} \in \mathcal{R}^{N}$ be such that for all $i \in S \cup T, R_{i}^{\prime \prime}=R_{i}^{\prime}$, and for all $i \in V, p\left(R_{i}^{\prime \prime}\right) \in \angle(c b a) \cap \operatorname{int} A$. Then by construction we have for all $i \in S$, $a P_{i}^{\prime \prime} c P_{i}^{\prime \prime} b$, for all $i \in T, c P_{i}^{\prime \prime} a I_{i}^{\prime \prime} b$, and for all $i \in V, c P_{i}^{\prime \prime} b P_{i}^{\prime \prime} a$. In particular we have $R^{\prime \prime}\left|\{a, b\}=R^{\prime}\right|\{a, b\}=R \mid\{a, b\}$. By $a, c \in \operatorname{int} A$ and $a P_{1}^{\prime \prime} c$, Case 1 yields $\neg c f\left(R^{\prime \prime}\right) a$. By weak Pareto, $\neg b f\left(R^{\prime \prime}\right) c$. Thus, by transitivity of $f\left(R^{\prime \prime}\right), \neg b f\left(R^{\prime \prime}\right) a$. Hence, by independence of irrelevant alternatives, $f\left(R^{\prime \prime}\right)|\{a, b\}=f(R)|\{a, b\}$ and $\neg b f(R) a$, the desired conclusion.

Case 3: $a \in A \backslash \operatorname{int} A$ and $b \in \operatorname{int} A$.

By taking $c$ close to $b$ in the interior of $A$ such that $\triangle(a, b, c)$ is rightangeled at $c$ and $h$ is closer to $b$ than to $c$ and observing that $\angle(b a c) \cap \operatorname{int} A \neq \emptyset$ because $h \in \angle((b a) c)$ is on the boundary, we deduce similarly to Case 2 that $\neg b f(R) a$.

Case 4: $a, b \in A \backslash \operatorname{int} A$.

Because $A \subseteq \operatorname{cl}(\operatorname{int} A)$, we can find $c \in \operatorname{int} A$ such that $\|c-a\|<\|c-b\|$. By Cases 2 and 3 and transitivity of $f\left(R^{\prime}\right)$ it follows that $\neg b f\left(R^{\prime}\right) a$. Hence, by $(2), \neg b f(R) a$, the desired conclusion. 


\section{Conclusion}

In this paper we solve a classical open problem in Arrovian social choice. For a onedimensional policy space and the domain of single-peaked preferences, we characterize the social welfare functions satisfying weak Pareto and independence of irrelevant alternatives. As a corollary we obtain that majority voting is transitive if and only if the number of individuals is odd and individual preferences are strictly quasi-concave.

For the case when preferences are Euclidean and the set of alternatives is $\mathbb{R}^{m}$ or $\mathbb{R}_{+}^{m}$ (where $m \geq 2$ ), Arrow's theorem has been previously established. However, these proofs do not apply for instance to the triangle with endpoints $(0,0),(1,0)$, and $(0.5,0.1)$. Then it is not possible to find a point $x$ belonging to this triangle such that $(0,0), x$, and $(1,0)$ are a free triple. We are the first to show Arrow's theorem when the set of alternatives is compact and convex. Here, we could have followed another classical method of proof. From a social welfare function satisfying weak Pareto and independence of irrelevant alternatives we could have constructed a twoagents social choice function $C$ with compact and convex agenda domain as follows: fix two coalitions $S$ and $N \backslash S$ (where $S \neq \emptyset$ and $S \neq N$ ) and consider only profiles where all agents belonging to $S$ have the same preference relation and all agents belonging to $N \backslash S$ have the same preference relation. For each compact and convex agenda $A^{\prime}$ and each profile $\left(R_{S}, R_{N \backslash S}\right) \in \mathcal{R} \times \mathcal{R}$ (here $S$ denotes "agent 1 " and $N \backslash S$ "agent 2"), let $C$ choose the quasi bliss point of $f\left(R_{S}, R_{N \backslash S}\right) \mid\left[p\left(R_{S} \mid A^{\prime}\right), p\left(R_{N \backslash S} \mid A^{\prime}\right)\right]$. Since at profiles with only two peaks, the weak Pareto set in $A^{\prime}$ is the line segment $\left[p\left(R_{S} \mid A^{\prime}\right), p\left(R_{N \backslash S} \mid A^{\prime}\right)\right]$ it is easy to see that $C$ satisfies weak Pareto, Arrow's choice axiom, and independence of infeasible alternatives. Then we could apply Duggan's (1996) theorem for spatial environments saying that $C$ must be dictatorial with dictator $S$ or $N \backslash S$. However, Duggan's theorem requires the preference domain to contain non-Euclidean preferences and our impossibility result does not. 


\section{References}

Arrow, K.J., 1951, Social Choice and Individual Values, John Wiley Press, New York.

Arrow, K.J. (1959): "Rational Choice Functions and Orderings," Economica 26:121127.

Arrow, K.J., 1963, Social Choice and Individual Values, 2nd ed., John Wiley Press, New York.

Barberà, S., F. Gul, and E. Stacchetti (1993): "Generalized Median Voter Schemes and Committees," Journal of Economic Theory 61 262-289.

Black, D. (1948): "On the Rationale of Group Decision Making," Journal of Political Economy 56:23-34.

Border, K.C. (1984): "An Impossibility Theorem for Spatial Models," Public Choice 43:293-305.

Border, K.C., and J.S. Jordan (1983): "Straightforward Elections, Unanimity and Phantom Voters," Review of Economic Studies 50:153-170.

Bossert, W. and J.A. Weymark (1993): "Generalized Median Social Welfare Functions," Social Choice and Welfare 10:17-33.

Campbell, D.E. (1993): "Euclidean Individual Preference and Continuous Social Preference," European Journal of Political Economy 9, 541-550.

Duggan, J. (1996): "Arrow's Theorem in Public Good Environments with Convex Technologies," Journal of Economic Theory 68:303-318.

Dutta, B., M.O. Jackson, and M. Le Breton (2001): "Strategic Candidacy and Voting Procedures," Econometrica 69:1013-1037.

Ehlers, L. (2001): "Independence Axioms for the Provision of Multiple Public Goods as Options," Mathematical Social Sciences 41:239-205. 
Ehlers, L., and J.A. Weymark (2001): "Candidate Stability and Nonbinary Social Choice," Working Paper, Vanderbilt University.

Kalai, E., E. Muller, and M.A. Satterthwaite (1979): "Social Welfare Functions when Preferences are Convex, Strictly Monotonic, and Continuous," Public Choice $34: 87-97$.

Kim, K.H. and F.W. Roush (1984): "Non Manipulability in Two Dimensions," Mathematical Social Sciences 8, 29-43.

Le Breton, M. and J.A. Weymark, 1996, An Introduction to Arrovian Social Welfare Functions on Economic and Political Domains, in "Social Choice and Political Economy" (Norman Schofield ed.), Kluwer, Dordrecht/Norwell, MA, 25-61.

Le Breton, M. and J.A. Weymark, 2000a, Arrovian Social Choice Theory on Economic Domains, Handbook of Social Choice and Welfare, K.J. Arrow, A.K. Sen, and K. Suzumura ed., North-Holland, forthcoming.

Le Breton, M. and J.A. Weymark (2000b): "Social Choice with Analytic Preferences," Social Choice and Welfare, forthcoming.

Moulin, H. (1980): “On Strategy-Proofness and Single-Peakedness," Public Choice $34: 437-455$.

Moulin, H. (1984): "Generalized Condorcet-Winners for Single-Peaked Preferences and Single-Plateaued Preferences," Social Choice and Welfare 1:127-147.

Peters, H., H. van der Stel, and T. Storcken (1992): "Independence of Irrelevant Alternatives and Strategy-Proofness on Economic Domains," in Game Theory and Economic Applications, Proceedings, New Delhi, India, December 1990, B. Dutta, D. Mookherjee, T. Parthasarathy, T.E.S. Ragahavan, D.Ray, and S.Tijs (ed.), Lecture Notes in Economics and Mathematical Systems Springer-Verlag Berlin Heidelberg 1992. 
Satterthwaite, M. (1975): "Strategy-Proofness and Arrow's Conditions: Existence and Correspondence Theorem for Voting Procedures and Social Choice Functions," Journal of Economic Theory 10:187-217. 


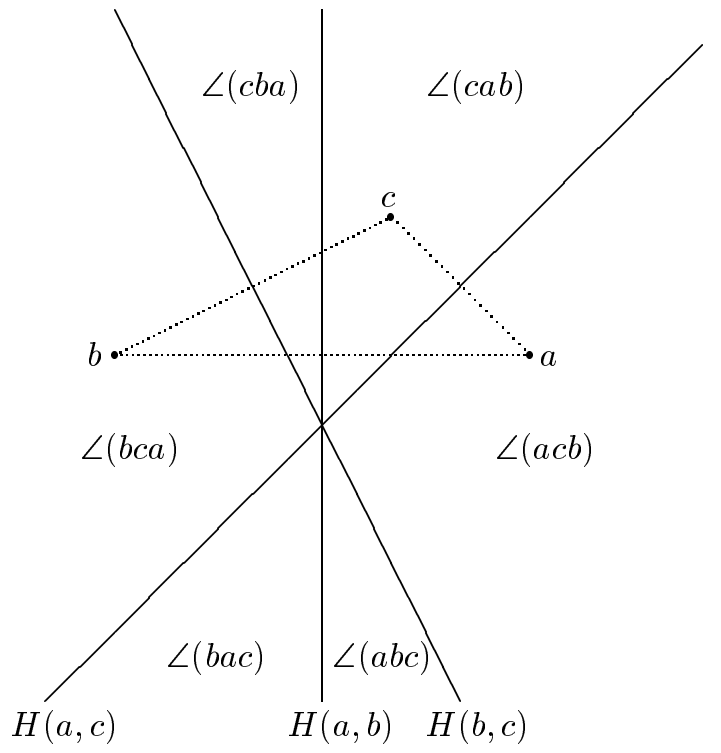

Figure 1: Illustration of the sets $\angle(a b c), \angle(a c b), \angle(b a c), \angle(b c a), \angle(c a b)$, and $\angle(c b a)$ for non-colinear $a, b$, and $c$ in $\mathbb{R}^{2}$.

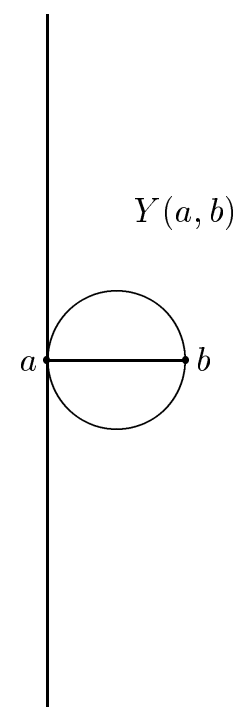

Figure 2: If $A=\mathbb{R}^{2}$, then $Y(a, b)$ is the half space right of the perpendicular to $[a, b]$ through $a$ excluding the set $\bigodot\left(\frac{1}{2}(a+b), \frac{1}{2}\|a-b\|\right)$. 